\title{
Synthesis of organic compounds in the circumstellar environment
}

\author{
Sun Kwok \\ Department of Physics, University of Hong Kong, Hong Kong, China \\ email: sunkwok@hku.hk
}

\begin{abstract}
Through the techniques of millimeter-wave and infrared spectroscopy, over 60 species of gas-phase molecules and a variety of inorganic and organic solids have been detected in the short phase of stellar evolution between the asymptotic giant branch and planetary nebulae. The chemical pathways that lead to the synthesis of complex organic compounds in such low-density environments are therefore important topics of astrochemistry. In this review, we summarize the observational evidence for the existence of complex aliphatic and aromatic compounds in these circumstellar environments, and discuss the nature of their possible carriers. Also discussed are a number of unidentified emission features which may also have an organic origin. The possible relations between these circumstellar organic matter with Solar System organic matter are explored.
\end{abstract}

Keywords. Stars: AGB and post-AGB, Planetary nebulae: general, ISM: molecules, ISM: lines and bands, infrared: ISM

\section{Introduction}

Nucleosynthesis of the element carbon and its dredge up to the photosphere represents the beginning of organic synthesis in the late stages of stellar evolution. The low surface temperature of aymptotic giant branch (AGB) stars allow the formation of gas-phase molecules such as $\mathrm{CO}, \mathrm{CN}$ and $\mathrm{C}_{2}$ in the photosphere. The onset of mass loss in the late AGB stage leads to the large-scale formation of a variety of gas-phase molecules in the stellar wind, including many organic molecules (Ziurys, these proceedings). Through $\mathrm{mm}$, submm, and infrared spectroscopy, over 60 different molecular species have been identified in the stellar winds of AGB stars. These molecules, and the solid particles that condense out of them, represent a major source of organic compounds in the Galaxy. The detection of presolar grains in meteorites originating from AGB stars establishes a direct link between stars and the Solar System, and AGB stars are a potential source of organic enrichment of the early Solar System (Zinner, these proceedings).

In this review, we summarize the observational evidence for the presence of organic solids in AGB stars, as well as their descendents proto-planetary nebulae (PPNe) and planetary nebulae (PNe). The rich and complex organic compounds synthesized in such a short $\left(10^{4} \mathrm{yr}\right)$ phase of stellar evolution establishes circumstellar environment as an important laboratory for the study of astrochemistry.

\section{Stellar synthesis of inorganic compounds}

The detection of the 9.7 and $18 \mu \mathrm{m} \mathrm{Si}-\mathrm{O}$ stretching and $\mathrm{Si}-\mathrm{O}-\mathrm{Si}$ bending modes of amorphous silicates in oxygen-rich AGB stars was the first realization that solids can efficiently form in the circumstellar environment of evolved stars (Woolf \& Ney 1969). In 
the IRAS sky survey, over 4000 O-rich and over 700 C-rich AGB stars have been found to possess the $9.7 \mu \mathrm{m}$ silicate feature and the $11.3 \mu \mathrm{m} \mathrm{SiC}$ feature, respectively (Kwok et al. 1997). ISO observations have detected a rich family of emission features attributed to cystalline silicates (olivine and pyroxine) in AGB stars and PNe (Jäger et al. 1998). A variety of refractory oxides (e.g., corunum, spinel) have also been seen in AGB stars (Posch et al. 1999). The detection of these substances in the circumstellar environment represents the beginning of the field astromineralogy (Henning 2003).

\section{Stellar synthesis of organic compounds}

The family of infrared emission features first discovered in the PN NGC 7027 by Russell et al. (1977) was identified as originating from stretching and bending modes of aromatic compounds (Duley \& Williams 1981). These features are not seen in AGB stars, suggesting that the carriers are either synthesized or only excited into emission after the end of the AGB phase. To understand the chemical synthesis history of these aromatic compounds, it would be useful to observe objects in transition between the AGB and PN stages. After the IRAS mission, an increasing number of such transition objects (often called "proto-PN" or "PPN") have been identified (Kwok 1993). ISO observations of these objects have found that they show the typical aromatic infrared bands (AIB), including the $\mathrm{C}-\mathrm{H}$ stretch at $3.3 \mu \mathrm{m}, \mathrm{C}-\mathrm{C}$ stretches at 6.2 and $7.7 \mu \mathrm{m}$, $\mathrm{C}-\mathrm{H}$ in-plane bending at $8.6 \mu \mathrm{m}$, and the $\mathrm{C}-\mathrm{H}$ out-of-plane bending mode at $11.3 \mu \mathrm{m}$. In addition, features attributed to aliphatic side chains attached to the rings, such as the $3.4 \mu \mathrm{m} \mathrm{C}-\mathrm{H}$ stretches and the $6.9 \mu \mathrm{m} \mathrm{C}-\mathrm{H}$ in-plane bending modes arising from the methyl $\left(-\mathrm{CH}_{3}\right)$ and methylene $\left(-\mathrm{CH}_{2}\right)$ groups attached to the aromatic rings (Geballe et al. 1992, Hrivnak et al. 2007, Figure 2). Also observed in the PPN spectra are broad emission plateau features around 8 and $12 \mu \mathrm{m}$, which can be interpreted as arising from

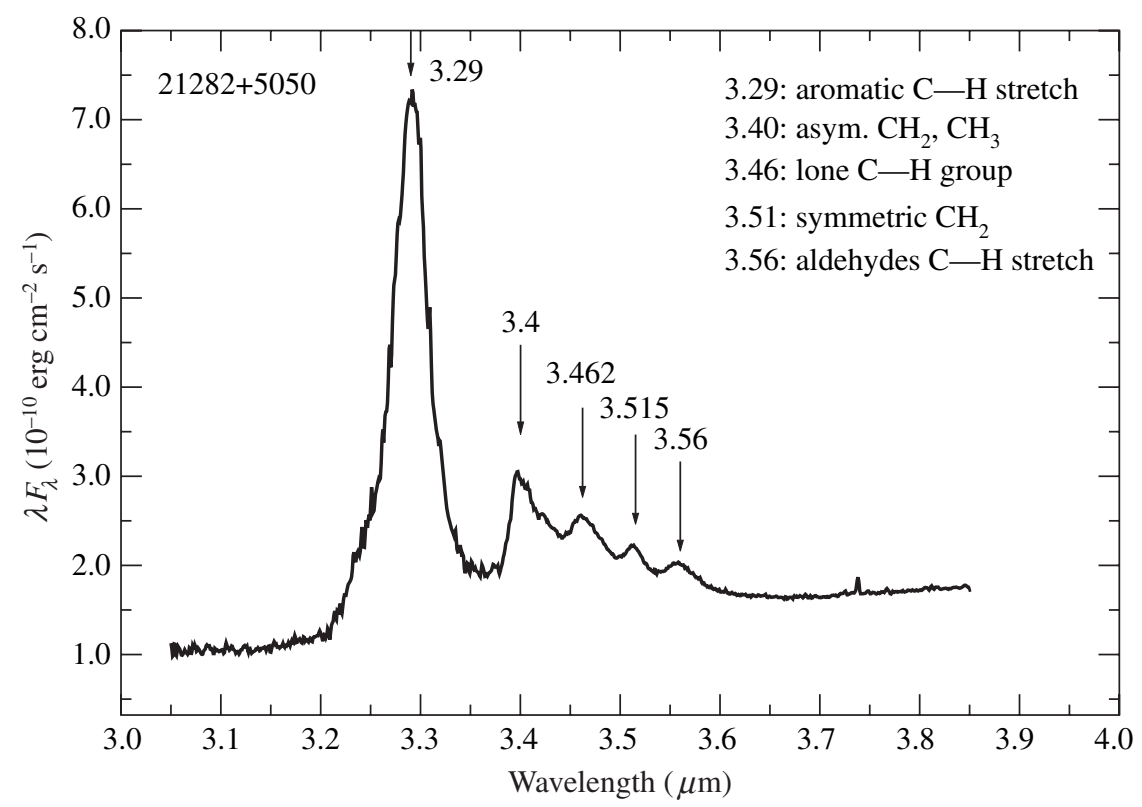

Figure 1. A spectrum of IRAS $21282+5050$ taken at the Keck Telescope showing the $3.4 \mu \mathrm{m}$ aliphatic features. The emission feature at $3.56 \mu \mathrm{m}$ could be due to an aldehyde group attached to the aromatic rings. 
the respective in-plane and out-of-plane bending modes of a mixture of aliphatic side groups (Kwok et al. 2001).

Another spectral distinction between $\mathrm{PN}$ and PPN is the presence of the other $\mathrm{C}-\mathrm{H}$ out-of-plane bending modes at $12.1,12.4$, and $13.3 \mu \mathrm{m}$ from aromatic rings with various degree of exposed edges (Witteborn et al. 1989, Kwok et al. 1999, Hony et al. 2001). In general, these features are seen in PPNe, and weak in PNe (Figure 2). These $\mathrm{C}-\mathrm{H}$ bending modes probably arise from a mixture of aromatic units in different configurations, examples of which can be seen in Hu \& Duley (2007).

The aromatic units can also undergo in-plane and out-of-plane distortion mode $\mathrm{C}-\mathrm{C}-\mathrm{C}$ vibrations which can lead to emission plateaus in the 15-20 $\mu$ m region (Van Kerkhoven et al. 2000). Such emission plateaus are seen in the spectrum of the young PN NGC 7027 (Figure 3).

A broad $(\sim 1 \mu \mathrm{m})$ emission feature at $15.8 \mu \mathrm{m}$ is seen in the Spitzer IRS spectra of the PPNe IRAS 06530-0213 and 23304+6147 (Hrivnak et al., these proceedings). Although quite prominent in these objects, this feature is not seen in PNe.

\section{Nature of the carriers of the infrared bands}

Unlike rotational transitions, the infrared features only tell us about the nature of the chemical bonds and the vibrational modes, but not the exact chemical structure of the carriers. All we can say is that the carrier is a carbonaceous compound with a mixture of aromatic and aliphatic structures. Example schematic of such structures can be found in Pendleton \& Allamandola (2002) and Kwok et al. (2001). The closest natural analogs of such structure is probably kerogen, which are random arrays of aromatic rings and

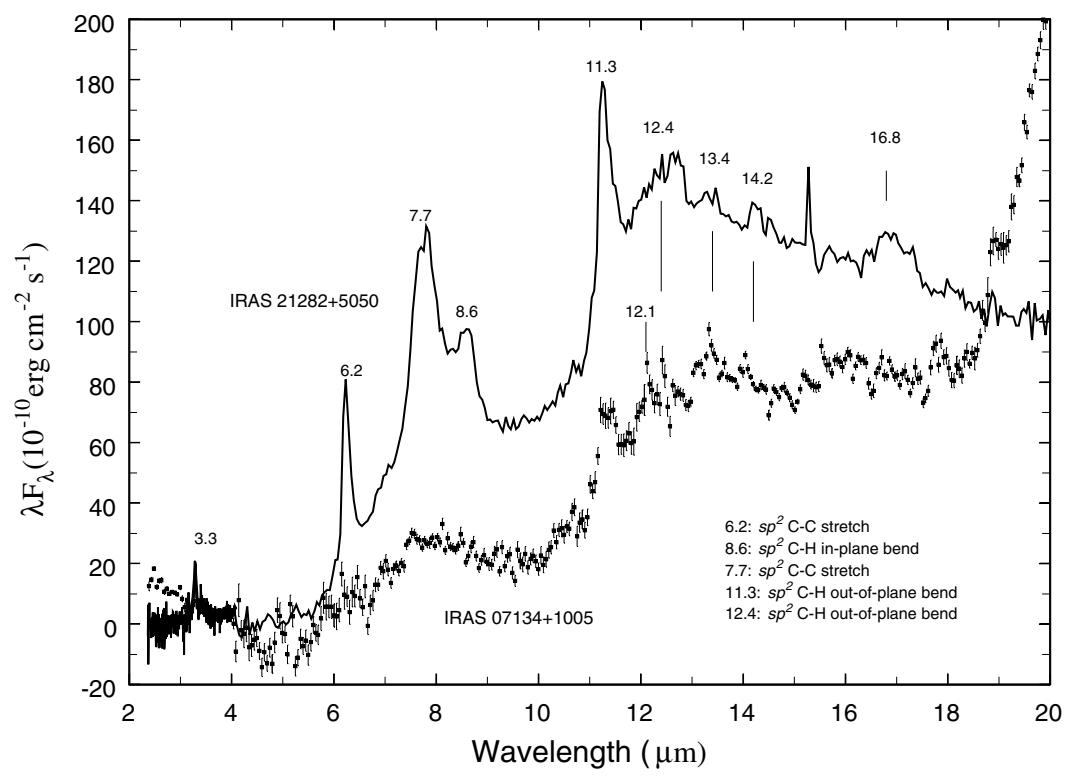

Figure 2. The ISO SWS01 spectra of the young PN IRAS $21282+5050$ and the PPN IRAS $07134+1005$, showing various aromatic C-H and C-C stretching and bending modes. The PPN spectra are characterized by the $12.1,12.4,13.3 \mu \mathrm{m}$ out-of-plane bending mode features from small aromatic units. 


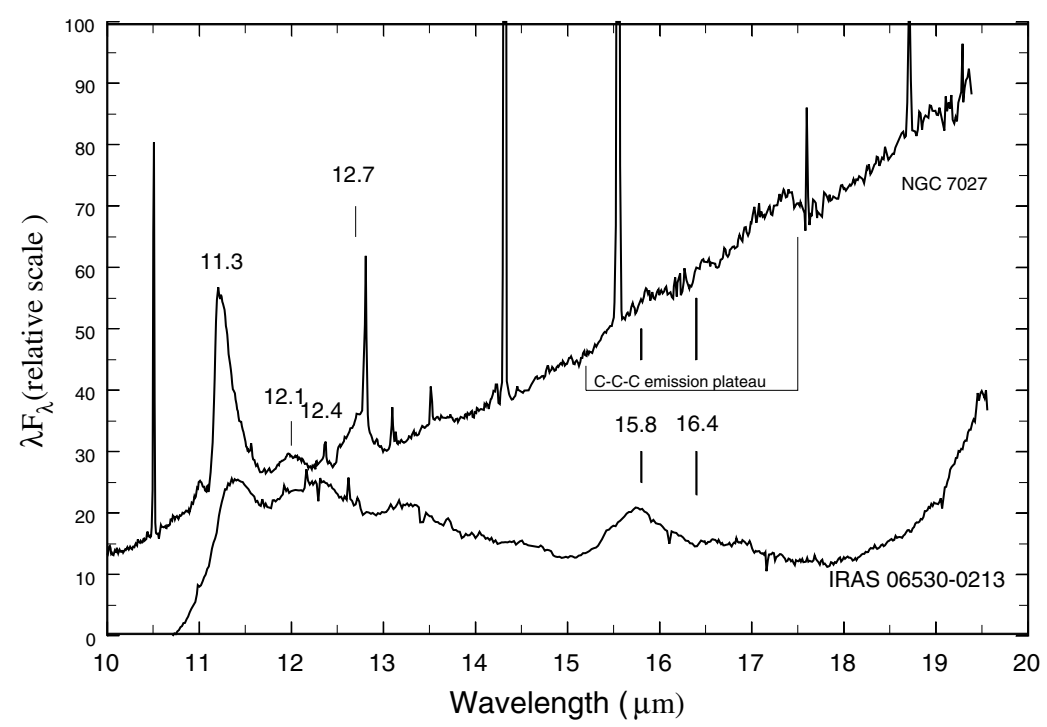

Figure 3. The Spitzer IRS spectra of young PN NGC 7027 and the PPN IRAS 06530-0213 showing 11.3, $12.1 \mu \mathrm{m}$ AIB features and the unidentified $15.8 \mu \mathrm{m}$ feature. The $15-17.5 \mu \mathrm{m}$ emission plateau in NGC 7027 could be due to $\mathrm{C}-\mathrm{C}-\mathrm{C}$ vibrational modes. The narrow feature in the NGC 7027 are atomic lines.

aliphatic chains with functional groups made up of $\mathrm{H}, \mathrm{O}, \mathrm{N}$, and $\mathrm{S}$ attached (Papoular et al. 1989, Papoular et al. 1996). In particular, the relative strengths of the 3.3 and $3.4 \mu \mathrm{m}$ feature and the 8 and $12 \mu \mathrm{m}$ emission plateaus seen in PPNe exhibit close resemblence to that of kerogen (Guillois et al. 1996). Soot, which is formed by combustion of hydrocarbon molecules in a flame, has also been suggested as a possible natural analog of circumstellar carbonaceous dust. Since the chemical composition of soot is highly dependent on the initial mix of the burning gas, its structure is too indefinite to be tested for spectral comparison.

Artificial substances created in laboratory simulations offer several possible counterparts to the circumstellar carbonaceous materials. Examples include hydrogenated amorphous carbon (HAC) produced from laser ablation of graphite in a hydrogen atmosphere (Scott et al. 1997, Duley, these proceedings), quenched carbonaceous composites (QCC) made from the quenching of plasma of methane (Sakata et al. 1987, Wada, these proceedings), and carbon-based nanoparticles created by laser pyrolysis of gas-phase $\mathrm{C}_{2} \mathrm{H}_{4}$, $\mathrm{C}_{4} \mathrm{H}_{6}$ molecules (Herlin et al. 1998).

We should remember that circumstellar AIB emission features lie on top of a strong infrared continuum (see, e.g., the continuum in the spectrum of NGC 7027 in Figure 3). This featureless dust continuum is likely to be the remnants of the circumstellar dust left over from the AGB stage, as evolved carbon stars such as IRC+20216, AFGL 3068 and IRAS $21318+5631$ show such strong dust continuum. The carrier of this continuum has often been attributed to amorphous carbon because of its featureless properties. Less evolved AGB stars show the $11.3 \mu \mathrm{m} \mathrm{SiC}$ feature, but this gives away to a featureless dust emission in highly evolved carbon stars. Complex and disordered organic compounds such as kerogen and tholins also show a strong infrared continuum and it is conceivable 
that this continuum is provided by complex organic particles of $\sim 10^{3} \mathrm{C}$ atoms. Tholins is an artificial substance made from the irradiation of gaseous mixtures of nitrogen and methane (Khare, these proceedings). Since nitrogen is a common element in circumstellar gas molecules (including cyanopolyynes), it is possible that $\mathrm{N}$ is incorporated into the solid component of circumstellar grains.

\section{Unidentified features}

In addition to the aromatic and aliphatic infrared features, AGB stars, PPNe, and PNe show other strong emission features which origins are not currently understood.

The 21- $\mu \mathrm{m}$ feature. The discovery of this very strong emission feature (Figure 4) in carbon-rich PPNe was a surprise (Kwok et al. 1989). Now over a dozen PPNe (but not AGB stars and PNe) have been seen to possess this feature (Hrivnak et al., these proceedings). Many different candidates have been proposed as the carrier of this feature, including the recent proposals of SiC (Speck \& Hofmeister 2004) and FeO (Posch et al. 2004).

The 30- $\mu \mathrm{m}$ feature. A broad emission feature peaking around $30 \mu \mathrm{m}$ is commonly observed in carbon-rich AGB stars, PPNe, and PNe (Figure 5). A significant fraction of the total energy output of PPNe and PNe can be emitted through this feature (Hrivnak et al. 2000).

Extended red emission. The extended red emission (ERE) is a broad emission band first detected in HD 44179 (the Red Rectangle), and is also seen in PNe (e.g., NGC 7027). It is believed to arise from photoluminescence of a semiconductor. A variety of inorganic (silicon nanoparticles, Witt et al. 1998; nanodiamonds, Chang et al. 2006) and organic (HAC, Duley 1985; QCC, Sakata et al. 1992) solids have been suggested as possible carriers.

The exact relationship between the above features and the AIB phenomenon is not clear, although carbon-rich association is assumed to be a common factor. All $21-\mu \mathrm{m}$

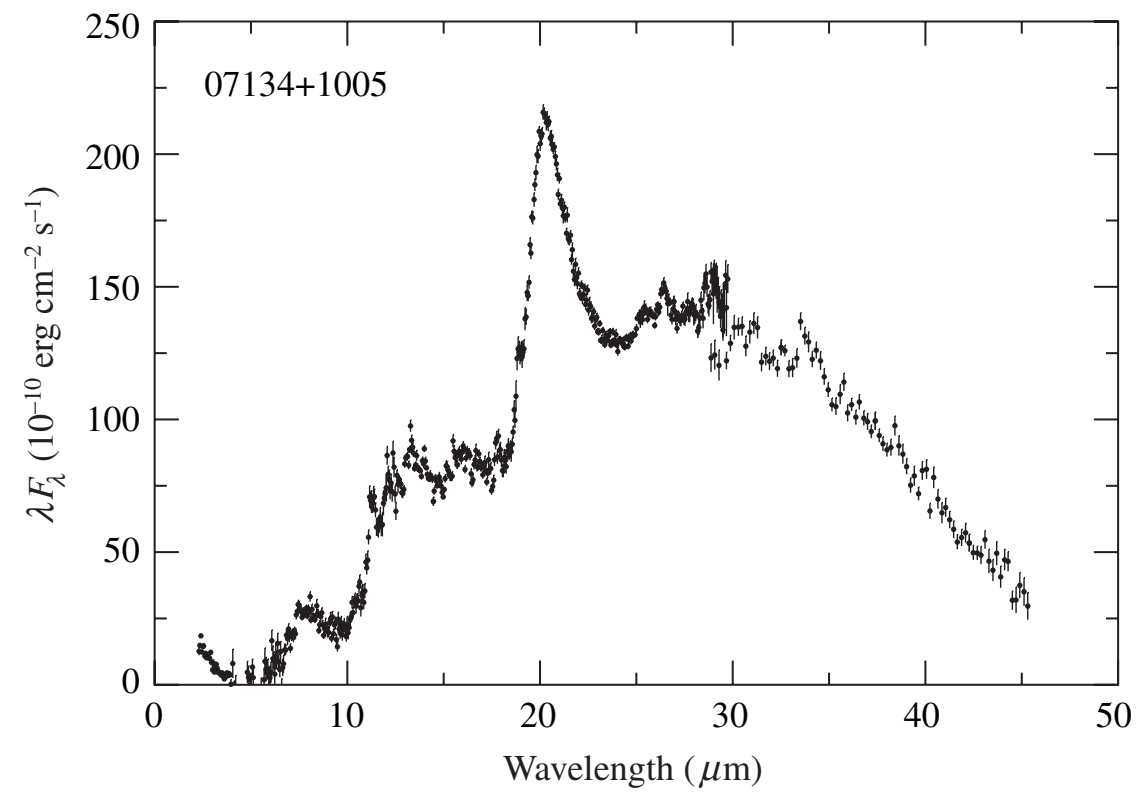

Figure 4. ISO SWS spectrum of the PPN IRAS 07134+1005 showing the strong unidentified $21 \mu \mathrm{m}$ emission feature. 


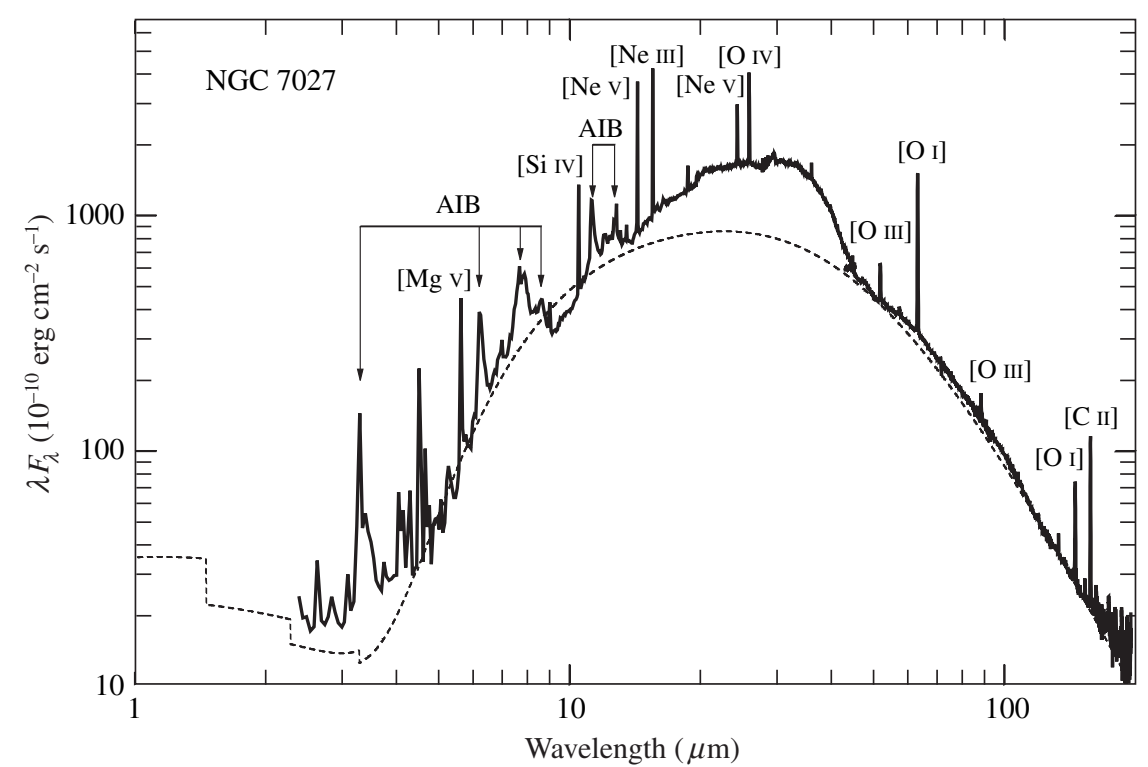

Figure 5. The ISO $S W S / L W S$ spectrum of NGC 7027 between 1-200 $\mu \mathrm{m}$. Some of the stronger atomic lines and AIB features are marked. The dashed line is a model fit composed of a sum of dust emission, $f$ - $b$ and $f$ - $f$ gas emission. The strong $30 \mu \mathrm{m}$ feature can be clearly seen above the dust continuum.

sources show the $30 \mu \mathrm{m}$ feature, but not vice versa. The ERE, although widely seen in the diffuse interstellar medium, is not commonly seen in evolved stars. This can be attributed to the need for an UV background for photoluminescence. Our difficulty in the identification of these features can be traced to our lack of understanding of spectral behavior of nanoparticles, as the mobility of electrons in a small cluster is reduced in a nanoparticle in comparison to bulk materials. Because of boundary effects and the resulting loss in symmetry, some vibrational modes can be more infrared active than in large crystals of the same chemical composition ( $\mathrm{Li} 2004)$.

\section{Chemical evolution and the role of photochemistry}

The detection of organic compounds in the ejecta of evolved stars gives us important information on how these species are formed. The evolution from AGB to PPN to PN is very short, and this gives us precise knowledge on the time scale of chemical synthesis. Since the AIB features first emerge in the PPN phase, what are the steps leading to the formation of ring molecules? Acetylene $\left(\mathrm{C}_{2} \mathrm{H}_{2}\right)$, believed to be the first building block of benzene, is commonly detected in evolved carbon stars through its $\nu_{5}$ fundamental band at $13.7 \mu \mathrm{m}$. Polymerization of $\mathrm{C}_{2} \mathrm{H}_{2}$ leads to the formation of diacetylene $\left(\mathrm{C}_{4} \mathrm{H}_{2}\right)$ and triacetylene $\left(\mathrm{C}_{6} \mathrm{H}_{2}\right)$ in PPN, cumulating in the formation of benzene (Cernicharo et al., 2001). The weakening of the 3.4 and $6.9 \mu \mathrm{m}$ from PPNe to PNe suggests a change from aliphatic to aromatic structures. This could be the result of photochemistry where the onset of UV radiation modify the aliphatic side groups through isomerizations, bond migrations, cyclization and ring closures and transform them into ring systems (Kwok et al. 2001). Hydrogen loss can also result in fully aromatic rings that are more stable than alkanes or alkenes. Evidence for such $\mathrm{H}$ loss can also be found in the weakening of the 12.1, 12.4, and 13.3- $\mu \mathrm{m}$ features and the strengthening of the $11.3-\mu \mathrm{m}$ feature from $\mathrm{PPNe}$ to PNe. The weakening of the aliphatic component due to photo-processing is also 
supported by an observed correlation between the central wavelengths of the 7.7 and $11.3 \mu \mathrm{m}$ features with the temperature of the central star (Sloan, these proceedings).

\section{Connection with the Solar System}

The connection between inorganic solids produced in AGB stars and the Solar System was established by the discovery of presolar grains in meteorites (Zinner, these proceedings; Nittler, these proceedings). It is clear that $\mathrm{SiC}$ and other refractory oxide grains produced by AGB stars have been able to find their way across the Galaxy and be incorporated into the constituents of the early Solar System. The $3.4 \mu \mathrm{m}$ aliphatic feature seen in PPNe are also seen in the spectra of interplanetary dust and comets (Flynn et al. 2003). The insoluble organic matter (IOM) in meteorites, comets, and interplanetary dust is mostly macromolecular and is similar to kerogen in structure (Kerridge 1999, Cruikshank, these proceedings). The $\mathrm{D} / \mathrm{H}$ and ${ }^{15} \mathrm{~N} /{ }^{14} \mathrm{~N}$ ratios seen in $\mathrm{IOM}$ are closer to interstellar values than Solar System values (Messenger 2000, Alexander, these proceedings). Is it possible that IOM is also stellar in origin?

In Section 4, we have noted the spectral similarities between the infrared spectra of PPN and kerogen. Terrestrial kerogen is biological in origin and produced by prolonged geological processing. If the carrier of the AIB features in PPNe is indeed similar to kerogen, then chemical synthesis of such complex organic compound is much more efficient in the circumstellar environment, which accomplishes the synthesis in thousands of years under extremely low density conditions.

\section{Conclusions}

We have shown that stars in the late stages of evolution are prolific producers of complex organic compounds. Through stellar winds, these organic materials are ejected to the interstellar medium and spread throughout the Galaxy (Kwok 2004). Although we are uncertain how much of these materials are destroyed or further processed in the interstellar medium, we do know at least some of the inorganic grains made to the early Solar System and were incorporated in meteorites. It is possible that stellar organic matter also contributed to the organic content in asteroids, comets, meteorites, and interplanetary dust particles.

The unidentified emission features seen in evolved stars may provide valuable clues to possible new organic compounds that can be synthesized under unusual physical conditions. These materials have the potential of broadening our understanding of organic chemistry beyond the terrestrial environment.

\section{Acknowledgements}

The work was supported by a grant from the Research Grants Council of the Hong Kong Special Administrative Region, China (Project No. HKU 7028/07P).

\section{References}

Cernicharo, J., Heras, A. M., Tielens, A. G. G. M., Pardo, J. R., Herpin, F., Guélin, M., \& Waters, L. B. F. M. 2001, ApJ (Letters), 546, L123

Chang, H.-C., Chen, K., \& Kwok, S. 2006, ApJ (Letters), 639, L63

Duley, W. W. 1985, MNRAS, 230, 1P

Duley, W. W. \& Williams, D. A. 1981, MNRAS, 196, 269

Flynn, G. J., Keller, L. P., Feser, M., Wirick, S., \& Jacobsen, C. 2003, Geochim. Cosmochim. Acta, 67, 4791 
Geballe, T. R., Tielens, A. G. G. M., Kwok, S., \& Hrivnak, B. J. 1992 ApJ (Letters), 387, L89 Guillois, O., Nenner, I., Papoular, R., \& Reynaud, C. 1996, ApJ, 464, 810

Henning, Th. (ed.), 2003, Astromineralogy (Springer)

Herlin, N., Bohn, I., Reynaud, C., Cauchetier, M., Galvez, A., \& Rouzaud, J.-N. 1998, A\&A, 330,1127

Hony, S. et al. 2001, A\&A, 370, 1030

Hrivnak, B. J., Volk, K., \& Kwok, S. 2000, ApJ, 535, 275

Hrivnak, B. J., Geballe, T. R., \& Kwok, S. 2007, ApJ, 662, 1059

Hu, A. \& Duley, W. W. 2007, ApJ (Letters), 660, L137

Jäger, C. et al. 1998, A\&EA, 339, 904

Kerridge, J. F. 1999, Space Sc. Rev., 90, 275

Kwok, S. 1993, Ann. Rev. Astr. Ap., 31, 63

Kwok, S. 2004, Nature, 430, 985

Kwok, S., Volk, K., \& Bernath, P. 2001, ApJ (Letters), 554, L87

Kwok, S., Volk, K., \& Bidelman, W. P. 1997, ApJS, 112, 557

Kwok, S., Volk, K., \& Hrivnak, B. J. 1989, ApJ (Letters), 345, L51

Kwok, S., Volk, K., \& Hrivnak, B. J. 1999, A\&A (Letters), 350, L35

Li, A. 2004, in: A. N. Witt, G. C. Clayton, \& B. T. Draine (eds.), Astrophysics of Dust, Vol. 309, p. 417

Messenger, S. 2000, Nature, 404, 968

Papoular, R., Conard, J., Giuliano, M., Kister, J., \& Mille, G. 1989, A\&A A, 217, 204

Papoular, R., Conard, J., Guillois, O., Nenner, I., Reynaud, C., \& Rouzaud, J.-N. 1996, A\& A, 315,222

Pendleton, Y. J. \& Allamandola, L. J. 2002, ApJS, 138, 75

Posch, Th., Mutschke, H., \& Andersen, A. 2004, ApJ, 616, 1167

Posch, T., Kerschbaum, F., Mutschke, H., Fabian, D., Dorschner, J. \& Hron, J. 1999, A\&6A, 352, 609

Russell, R. W., Soifer, B. T., \& Willner, S. P. 1977, ApJ (Letters), 217, L149

Sakata, A., Wada, S., Onaka, T., \& Tokunaga A.T. 1987, ApJ (Letters), 320, L63

Sakata, A., Wada, S., Narisawa, T. et al. 1992, ApJ (Letters), 393, L83

Scott, A. D., Duley, W. W., \& Jahani, H. R. 1997, ApJ (Letters), 490, L175

Speck, A. K. \& Hofmeister, A. N. 2004, ApJ, 600, 986

Van Kerckhoven, C., et al. 2000, A\& A, 357, 1013

Witt, A. NJ., Gordon, K. D., \& Furton, D. G. 1998, ApJ (Letters), 501, L111

Woolf, N. J. \& Ney, E. P. 1969. ApJ (Letters), 155, L181

\section{Discussion}

Pendleton: Do you think that stars made aliphatic molecules first and then they become aromatic? Some people said that it might go the other way that you might have aromatic molecules and they break apart to shorter chains.

Kwok: The first organic compounds that are synthesized are probably very amorphous, dirty, and complicated. Benzene is probably the first to form, with further rings gradually added. In the process, all kinds of side chains got attached to these basic aromatic units. This is just not a clean chemistry. By the time that the peripheral materials got cleaned up by photo processes, the aromatic clusters then link together and become larger and larger.

ZIURYS: What fraction of these organic compounds do you think contain atoms other than carbon and hydrogen, e. g., oxygen and nitrogen?

Kwok: One thing I am certain is that these compounds will very likely contain impurities. For example, one carbon atoms in the aromatic rings may be substituted by 
nitrogen. This will result in a change in the frequency of the vibrational modes. However, at present we do not have the sensitivity nor spectral resolution to have confirmed detection of such species.

Athena: Can you say a little bit about the interpretation of the ERE?

Kwok: The ERE was discovered in the Red Rectangle and has been seen in planetary nebulae, for example NGC 7027. They have been suggested to originate from HAC, QQC, $\mathrm{C}_{60}$, silicon nanoparticles, etc., as well as from nanodiamonds as we proposed in a paper in 2006. The only thing we are certain is that it is a photoluminescence phenomenon from a semiconductor, most likely a carbonaceous one. ERE may be one of the manifestation of carbonaceous organic compounds that we are discussing here.

SANDFORD: I would like to make a comment about the issue of aliphatic versus aromatic. Most of the discussions on aliphatics have been about side groups hanging off the edges of aromatic domains, but in fact there is the additional possibility that the aromatic grains can have extra hydrogen atoms on some of the peripheral carbons. In this case, you have a hybrid molecule which spectrum looks like aromatic plus aliphatic. With UV photolysis you don't have to build new rings - you just need to knock a hydrogen off one of the aliphatic rings and it starts to become more aromatic.

MATHEws: Going back to the question about the aromatic and aliphatic being together, I think what we have are HCN polymers and they are not as easy to identify as aromatics. Since we have ammonia, methane and nitrogen which can give rise to HCN and acetylene. Acetylene will give you hydrocarbons and $\mathrm{HCN}$ will give you HCN polymers. I predict you always find the two together. I am glad you emphasize that further observational techniques are necessary before we actually see the HCN polymers in the circumstellar envelopes.

KWOK: I think the presence of HCN polymers is very likely. However, my understanding is that they don't have strong spectroscopic signatures which make astronomical identification difficult. They may be widely present but we can't be sure that they are there.

HRIVNAK: We did a study to look at the strength of the $3.4 \mu \mathrm{m}$ feature compared to the $3.3 \mu \mathrm{m}$ feature and in general the 3.4 gets weaker as you go from PPNe to PNe. But there are some counterexamples.

Kwok: Qualitatively we know when a star evolves from low temperature to high temperature which corresponds to the changes in spectral type. But in practice since the progenitors of planetary nebulae have a variety of central stars mass they evolve at highly different rates, there may not be a strict one-to-one correspondence between chemcal evolution and spectral types. 


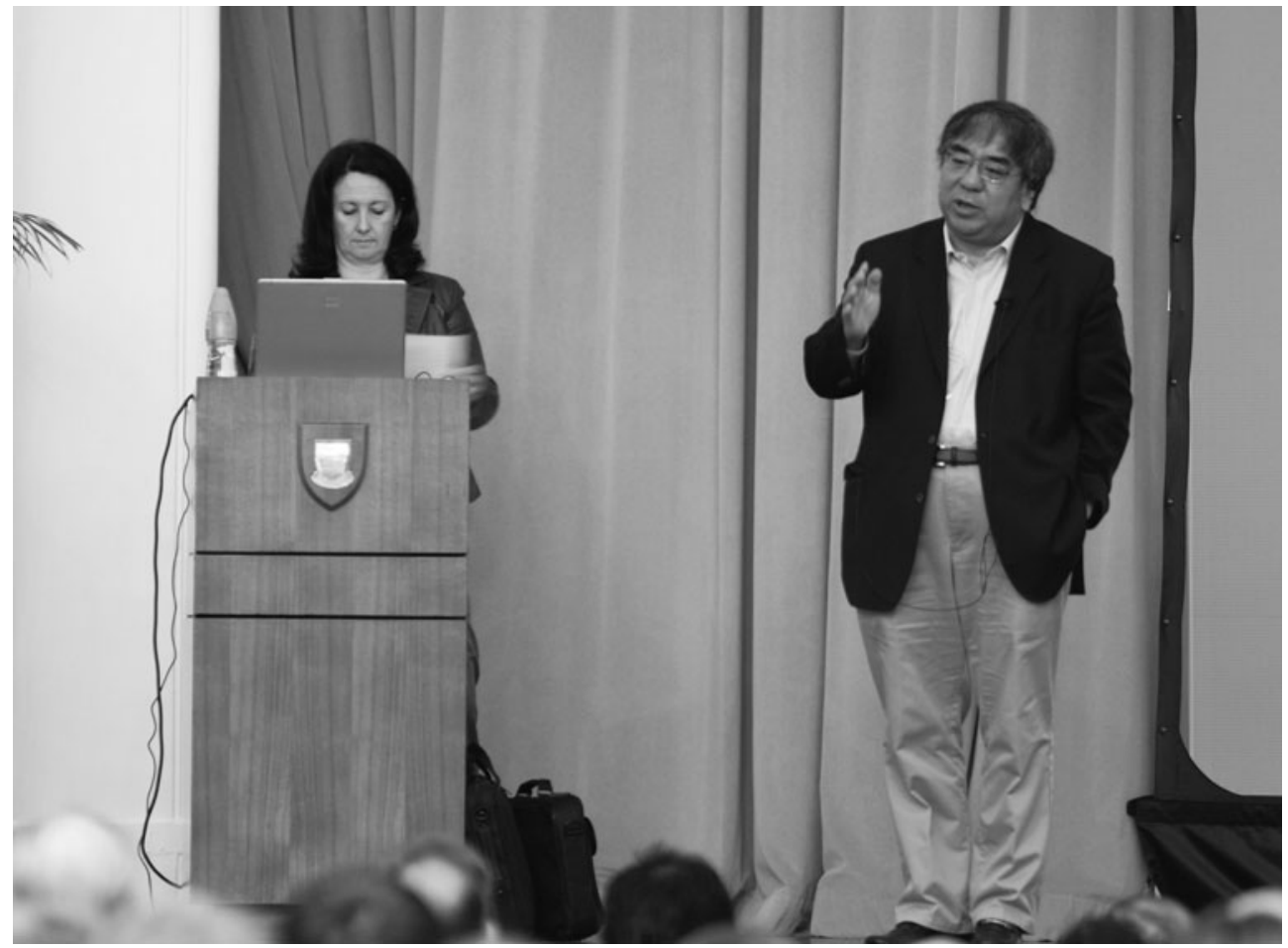

Sun Kwok answering questions after his talk (photo by Sze-Leung Cheung). 